\title{
Sublattice Magnetization and Néel Transition in the 2D Quantum Heisenberg Antiferromagnet
}

\author{
Eduardo C. Marino and Marcello B. Silva Neto ${ }^{\text {a } *}$ \\ anstituto de Física, Universidade Federal do Rio de Janeiro, Caixa Postal 68528, Rio de Janeiro - RJ, \\ 21945-910, Brazil
}

\begin{abstract}
We present an analytic expression for the finite temperature sublattice magnetization, at the Josephson scale, in two-dimensional quantum antiferromagnets with short range Néel order. Our expression is able to reproduce both the qualitative behaviour of the phase diagram $M(T) \times T$ and the experimental values of the Néel temperature $T_{N}$ for either doped $\mathrm{YBa}_{2} \mathrm{Cu}_{3} \mathrm{O}_{6.15}$ and stoichiometric $\mathrm{La}_{2} \mathrm{CuO}_{4}$ compounds.
\end{abstract}

It is the purpose of this work to show that the experimental data for the sublattice magnetization of $\mathrm{La}_{2} \mathrm{CuO}_{4}$ [1] and $\mathrm{YBa}_{2} \mathrm{Cu}_{3} \mathrm{O}_{6.15}$ [2] can in fact be described still in the context of a two-dimensional square-lattice quantum Heisenberg antiferromagnet at finite temperatures. Our starting point is the observation that the nature of the spin correlations in the renormalized classical regime is consistent with one of the three possibilities of fig. 1, according to the observation wave vector [3]. Next we argue that the spin dynamics in the intermediate Goldstone region can be described by an effective field theory for the low energy, long wavelength fluctuations of the spin fields about a state with short range Néel order. In fact, since at low $T$ the three regions of fig. (1) are well separated, dynamic scaling hypotesis is valid and a hydrodynamic picture in which short wavelength spin waves follow adiabatically the disordered background is applicable [4]. Finally, we show that the destruction of antiferromagnetic order in real materials can be associated with the collapse of the Goldstone region in fig. (1).

We start already in the continuum formulation of the problem by considering the partition function

$\mathcal{Z}(\beta)=\int \mathcal{D} n_{l} \delta\left(n_{l}^{2}-1\right) \exp \left(-\mathcal{I}\left(n_{l}\right)\right)$

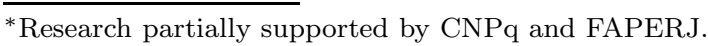

\section{RENORMALIZED CLASSICAL}

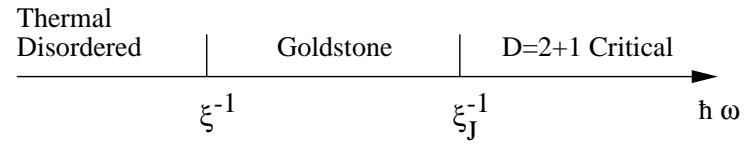

Figure 1. Properties of the $2 D$ quantum Heisenberg antiferromagnet as a function of the observation wave vector $k$, or frequency $\omega$.

where

$\mathcal{I}\left(n_{l}\right)=\frac{\rho_{0}}{2 \hbar} \int_{0}^{\hbar \beta} \mathrm{d} \tau \int \mathrm{d}^{2} \mathbf{x}\left[\left(\nabla n_{l}\right)^{2}+\frac{1}{c_{0}^{2}}\left(\partial_{\tau} n_{l}\right)^{2}\right]$

with $n_{l}=(\sigma, \vec{\pi}), l=1, \ldots, N=3$. Let us next construct an effective field theory for the low frequency, long wavelength fluctuations of the staggered components of spin-fields about a state with short range Néel order. To this end we perform integration of the Fourier components of the fields in (11) with frequency inside momentum shells $\kappa \leq|\vec{k}| \leq \Lambda$. The resulting partition function is such that, for large $N$, the leading contribution comes from the scale dependent stationary configurations $\left\langle n_{3}\right\rangle_{\kappa}$ and $\mathrm{i}\langle\lambda\rangle_{\kappa}=m_{\kappa}^{2}$, solutions of the saddle-point equation

$\left\langle n_{3}\right\rangle_{\kappa}^{2}=\frac{1}{g_{0}}-\frac{1}{\beta} \sum_{n=-\infty}^{\infty} \int_{\kappa}^{\Lambda} \frac{\mathrm{d}^{2} \mathbf{k}}{(2 \pi)^{2}} \frac{1}{\mathbf{k}^{2}+\omega_{n}^{2}+m_{\kappa}^{2}}$,

where, as usual, $\lambda$ is a Lagrange multiplier for the averaged fixed length constraint and $g_{0}=N / \rho_{0}$. 
Due to the presence of the IR cutoff $\kappa$, the system can be found in two different phases (regimes): ordered (asymptotically free) or disordered (strongly coupled); depending on the size of $\xi_{\kappa}=1 / \kappa$ relative to $\xi$ : smaller (high energies) or larger (low energies). In the ordered phase, $\xi_{\kappa} \ll \xi, m_{\kappa}=0$ is the solution that minimizes the free energy and the $2 D$ system is then characterized by a nonvanishing effective sublattice magnetization $\left\langle n_{3}\right\rangle_{\kappa} \neq 0$, a divergent effective correlation length $\xi_{\text {eff }}=1 / m_{\kappa}=\infty$ and gapless excitations in the spectrum.

We can subtract the linear divergence in (2) by the renormalization $1 / g_{0}=1 / g_{c}+\rho_{s} / 4 \pi N$, where $g_{c}=4 \pi / \Lambda$ and $\rho_{s}=(\hbar c / a) \sqrt{S(S+1 / 2)} / 2 \sqrt{2}$ is the bulk spin stiffness. Now, after momentum integration and frequency sum, we obtain the running spin stiffness

$\rho_{s}(\kappa, \beta)=\frac{\rho_{s}}{2}+\frac{N}{\beta} \ln (2 \sinh (\beta \kappa / 2))$.

It is clear from the above expression that at long distances, $k \rightarrow 0$, the system is found disordered and strongly coupled. No sublattice magnetization can be measured. Here, conversely, in order to obtain a finite temperature phase transition in the $2 D$ system, we will rather fix the scale $\kappa$ and study the behaviour of the spin stiffness (3) with the running parameter as being the temperature. For this it suffices to impose the boundary condition

$\rho_{s}(\kappa, T=0)=\rho_{s}$.

From (4) we conclude that $\kappa=\rho_{s} / N$, which is exactly the inverse Josephson correlation length $\kappa=\xi_{J}^{-1}$. This should not be surprising since the spin stiffness is itself a microscopic, short wavelength quantity defined at the Josephson scale. Now, inserting (4) in (3), the expression for the finite temperature effective sublattice magnetization, $M(T) \equiv \rho_{s}\left(\kappa=\rho_{s} / N, T\right)$, becomes

$M(T)=\frac{M_{0}}{2}+N T \ln \left(2 \sinh \left(\frac{M_{0}}{2 N T}\right)\right)$,

with $M_{0}=\rho_{s}$. As the temperature increases the sublattice magnetization $M(T)$ vanishes at a Néel temperature $T_{N}$ given by

$T_{N}=\frac{M_{0}}{N \ln 2}=\frac{\xi_{J}^{-1}}{\ln 2}$.

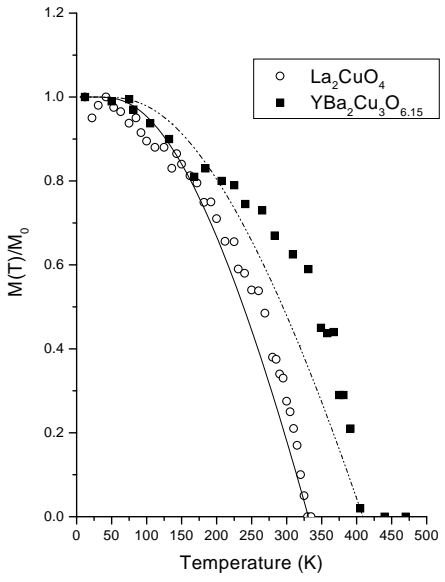

Figure 2. Relative sublattice magnetization at the Josephson scale for $\mathrm{La}_{2} \mathrm{CuO}_{4}$ (solid line and $\hbar c=0.78 \mathrm{eV} \AA$ ) and $\mathrm{YBa}_{2} \mathrm{Cu}_{3} \mathrm{O}_{6.15}$ (dash dot dot line and $\hbar c=0.90 \mathrm{eV} \AA$ ).

This also corresponds to the value of $T$ for which $\xi=\xi_{J}$ and the Goldstone region in fig. (1i) collapses.

In fig. (2) we plot $M(T) / M_{0}$ against experiment for either $\mathrm{La}_{2} \mathrm{CuO}_{4}$ and $\mathrm{YBa}_{2} \mathrm{Cu}_{3} \mathrm{O}_{6.15}$ and for $N=3$. To compute $\rho_{s}$ we have used $S=1 / 2$, $a=3.8 \AA$ and the experimental values of $c$. A more detailed analysis of the problem, with a discussion on how the actual deviation of the experimental points from the theoretical curves can be accounted for, can be found in [5].

\section{REFERENCES}

1. B. Keimer et al., Phys. Rev. B45, 7430 (1992).

2. J. M. Tranquada et al., Phys. Rev. Lett. 60, 156 (1988).

3. A. Chubukov et al., Phys. Rev. B49, 11919 (1994).

4. S. Chakravarty et al., Phys. Rev. B39, 2344 (1989).

5. E. C. Marino and M. B. Silva Neto, eprint archive cond-mat/9912143. 